\title{
Orbital Approach to the Electronic Structure of Solids
}

ENRIC CANADELL

Institut de Ciència de Materials de Barcelona (CSIC)

MARIE-LIESSE DOUBLET

CNRS - University of Montpellier

CHRISTOPHE IUNG

University of Montpellier 


\section{Contents}

\section{Elementary introduction to the transport}

properties of solids

1.1 Free electron model

1.1.1 One-dimensional system

1.1.2 Generalisation to a three-dimensional system

1.2 Conductivity of real solids

1.2.1 Factors influencing the conductivity

1.2.2 Band structure of real solids

1.2.3 Metallic behaviour

1.2.4 Semiconducting and insulating behaviour

1.2.5 Number of carriers

\section{Electronic structure of molecules: use of symmetry}

2.1 Molecular orbital theory

2.1.1 Born-Oppenheimer approximation

2.1.2 One-electron approximation

2.1.3 LCAO approximation

2.1.4 Secular equations and secular determinant

2.1.5 Basic features of the Hückel and extended Hïckel methods

2.1.6 Symmetry properties of the molecular orbitals

2.2 A short review of the theory of symmetry point groups 19

$\begin{array}{ll}\text { 2.2.1 Different symmetry point groups } & 19\end{array}$

$\begin{array}{ll}2.2 .2 \text { Classes } & 21\end{array}$

2.2.3 Basis for an irreducible representation $\quad 22$

2.3 Application to the study of the $\pi$ system of regular cyclobutadiene

2.3.1 Decomposition of the $\Gamma\left(p_{z}\right)$ basis 26

2.3.2 Determination of the basis elements for different irreducible representations

2.3.3 Molecular orbital diagram of the $\pi$ system of regular cyclobutadiene

2.4.1 Ligands and formal oxidation state 31

2.4.2 The $\mathrm{ML}_{6}$ octahedral complex 33

2.4.3 Distortions of a complex $\quad 39$ 


\section{Electronic structure of one-dimensional systems:} basic notions

3.1 Bloch and crystal orbitals $\quad 45$

3.1.1 Bloch orbitals $\quad 46$

3.1.2 Crystal orbitals $\quad 49$

3.2 Electronic structure of the model chain $\mathrm{H}_{n}$

3.2.1 Representation of the $\mathrm{CO}(\Gamma)$ and $\mathrm{CO}(\mathrm{X})$ functions 51

3.2.2 Energy of the crystal orbitals in the Hückel approach $\quad 52$

3.2.3 Band structure $\quad 54$

3.2.4 Basis for an energy level $E( \pm \vec{k}) \quad 55$

3.2.5 Fermi level of the $\mathrm{H}_{n}$ chain $\quad 57$

3.3 Electronic structure of the dimerised model chain $\left(\mathrm{H}_{2}\right)_{n^{\prime}} \quad 58$

3.3.1 Formal determination of the band structure 58

3.3.2 Qualitative determination of the band structure 61

3.4 Comparison of the regular $\mathrm{H}_{n}$ and dimerised $\left(\mathrm{H}_{2}\right)_{n^{\prime}}$ chains 63

3.4.1 Comparison of the band structures of the regular $\mathrm{H}_{n}$ chain generated by either a simple or a double unit cell 63

3.4.2 Dimerisation in the $\mathbf{H}_{n}$ chain: notion of distortion in a periodic system

\section{First-order Peierls distortions in periodic ID systems}

4.1 Analysis of the model system $\left(\mathrm{H}^{0.5+}\right)_{n}$

4.1.1 Effect of a tetramerisation on the Fermi level

4.1.2 Effect of a tetramerisation on the states near the Fermi level

4.1.3 Effect of a tetramerisation on the band structure

4.2 Analysis of first-order Peierls distortion in terms of a charge density wave

4.3 Nesting vector

4.4 Commensurate and incommensurate distortions 81

$\begin{array}{lll}\text { 4.4.1 Commensurate distortion } & 81\end{array}$

$\begin{array}{lll}\text { 4.4.2 Incommensurate distortion } & 83\end{array}$

$\begin{array}{lll}4.4 .3 \text { Comparison } & 83\end{array}$

$\begin{array}{lll}4.5 & \text { Conclusions } & 83\end{array}$

\section{Application to trans-polyacetylene 85}

$\begin{array}{lll}5.1 & \text { Electronic structure of ethylene } & 86\end{array}$

5.2 Main aspects of the band structure for trans-polyacetylene 87

5.3 Detailed analysis of the band structure of trans-polyacetylene $\quad 88$

5.4 Determination of the band structure of trans-polyacetylene using the fragment formalism

5.4.1 Calculation of the band structure by means of the Hückel approach 
6 Handling the symmetry in ID compounds 96

6.1 Analysis of the $\mathrm{A}_{n}$ system 96

6.1.1 Analysis of the cyclic $A_{n}$ system 96

6.1.2 Analysis of the linear $A_{n}$ system 101

6.1.3 Notion of group of a $k$ point 104

6.2 Application to the determination of the band structure for the $\mathrm{A}_{n}$
linear system, where $\mathrm{A}$ is an atom

6.2.1 Group of the different $k$ points 105

6.2.2 Symmetry of the different Bloch orbitals 105

$\begin{array}{ll}\text { 6.2.3 Bands associated with } \sigma \text {-type overlaps } & 107\end{array}$

6.2.4 Complete band structure 108

6.3 Band structure of the hypothetical $(\mathrm{NaCl})_{n}$ chain $\quad 109$

6.3.1 Group of the different $k$ points 110

6.3.2 Bands associated with $\sigma$-type overlaps $\quad 110$

6.3.3 Complete band structure 112

6.4 Consequences of the existence of a glide plane 113

6.4.1 Using point group symmetry properties in
trans-polyacetylene

6.4.2 Complete space group (non-symmorphic) of trans-polyacetylene 115

6.4.3 Crystal orbitals of trans-polyacetylene by means of the
non-symmorphic space group $G=T_{n} \otimes C_{2 h} \otimes\left\{E, g_{\sigma}\right\} \quad 117$

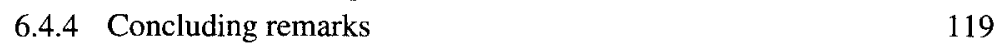

$\begin{array}{ll}6.5 & \text { Work plan for the study of a } 1 \mathrm{D} \text { system } \\ \end{array}$

7 Application to polyacene 122

$\begin{array}{ll}7.1 \text { Band structure near the Fermi level } & 123\end{array}$

$\begin{array}{ll}7.1 .1 & \text { Unit cell definition } \\ 7.123\end{array}$

7.1.2 Symmetry analysis of the chain $\quad 123$

$\begin{array}{ll}\text { 7.1.3 Appropriate fragment orbitals } & 123\end{array}$

7.1.4 Crystal orbitals at the $\Gamma$ and X points 124

7.1.5 $\pi$-type band structure of polyacene 126

$\begin{array}{ll}7.2 \text { Distortions in polyacene } & 128\end{array}$

$\begin{array}{ll}\text { 7.2.1 Disappearance of the } \sigma_{x y} \text { symmetry plane } & 128\end{array}$

$\begin{array}{ll}\text { 7.2.2 Disappearance of the } \sigma_{y z} \text { symmetry plane } & 128\end{array}$

$\begin{array}{ll}7.3 \text { General remarks concerning Peierls distortions } & 130\end{array}$

$\begin{array}{ll}\text { 7.3.1 First-order Peierls distortions } & 130\end{array}$

$\begin{array}{ll}\text { 7.3.2 Second-order Peierls distortions } & 131\end{array}$

8 Electronic structure of selected inorganic chains 133

$8.1 \mathrm{KCP} \quad 133$

8.1.1 Band structure of the eclipsed chain $\left[\mathrm{Pt}(\mathrm{CN})_{4}\right]^{(2-\delta)-} \quad 134$

8.1.2 Band structure of KCP (staggered chain) 139

$\begin{array}{ll}8.1 .3 \text { Conclusions } & 142\end{array}$

$8.2\left(\mathrm{ML}_{4} \mathrm{~L}^{\prime}\right)_{n}$ chains $\quad 143$

$\begin{array}{lll}\text { 8.2.1 Symmetry } & 143\end{array}$ 
8.2.2 Choice of the fragment orbitals to generate the Bloch orbitals

8.2.3 Analysis of the Bloch orbitals at the $\Gamma$ and $X$ points

8.2.4 Symmetry of the Bloch orbitals

8.2.5 Band structure

8.2.6 Study of the $\left(\mathrm{ReCl}_{4} \mathrm{~N}\right)_{n}$ chain

8.2.7 Electronic structure of the $\left(\mathrm{Pt}\left(\mathrm{NH}_{2} \mathrm{Et}_{4} \mathrm{Cl}^{2+}\right)_{n}\right.$ chain

\section{Electronic structure of 2D and 3D systems}

9.1 Basic concepts

9.1.1 Direct and reciprocal lattices 157

9.1.2 Bloch and crystal orbitals $\quad 159$

9.1.3 Brillouin zone
9.1.4 Symmetry and the Brillouin zone

9.2 Analysis of the electronic structure of 2D model systems

9.2.3 $\pi$-type band structure of hexagonal graphene layers $\quad 173$

\section{Density of states}

10.1 Calculation and analysis of the density of states

10.1.1 Density of states

10.1.2 Projected density of states

10.1.3 Crystal orbital overlap population

10.2 Combined use of DOS and COOP: electronic structure of the $\mathrm{MPS}_{3}$ layered phases

10.3 Step-by-step determination of the density of states: the $\left(\mathrm{Pt}\left(\mathrm{NH}_{3}\right)_{4} \mathrm{Cl}\right)^{2+}$ chain

10.4 Density of states and fragment molecular orbital interaction analysis: application to the $\left[\left(\mathrm{C}_{5} \mathrm{H}_{5}\right) \mathrm{M}\right]$ chains

10.5 Transition metal diborides with the $\mathrm{AlB}_{2}$ structure type: a 3D case study

\section{Fermi surface and low-dimensional metals}

11.1 Notion of Fermi surface

11.2 Nesting vector and electronic instabilities in low-dimensional metals

11.3 Monoclinic $\mathrm{TaS}_{3}$ versus $\mathrm{NbSe}_{3}$

11.3.1 Crystal structure and electron counting 211

11.3.2 Qualitative band structure

11.3.3 Qualitative Fermi surface: differences between $\mathrm{NbSe}_{3}$ and $\mathrm{TaS}_{3}$ 
11.4.1 Octahedral distortions and $t_{2 g}$ level splitting in $\mathrm{MoO}_{6}$ octahedra

11.4.2 $\mathrm{MoO}_{5}$ chain with corner-sharing octahedra: counting of $2 p$ oxygen antibonding contributions

11.4.3 $\mathrm{A}_{0.33} \mathrm{MoO}_{3}(\mathrm{~A}=\mathrm{K}, \mathrm{Rb}, \mathrm{Cs}, \mathrm{Tl}) 2 \mathrm{D}$ red bronzes: metallic or insulating?

11.4.4 $\mathrm{A}_{0.3} \mathrm{MoO}_{3}(\mathrm{~A}=\mathrm{K}, \mathrm{Rb}, \mathrm{Tl})$ blue bronzes: $2 \mathrm{D}$ solids with pseudo-1D behaviour

11.4.5 Looking for 1D systems where there seem to be none: the concept of hidden nesting

11.5 Low-dimensional molecular conductors

11.5.1 An archetypal molecular metal: (TMTSF) ${ }_{2} \mathrm{PF}_{6}$

11.5.2 Chemically modifying the electronic structure of molecular conductors

11.5.3 Structurally complex materials with simple band structures

11.5.4 A case study: 1D vs 2D character of the carriers in some $\alpha$ phases of BEDT-TTF

11.5.5 Electronic structure and folding: how to relate the band structure and Fermi surface of different salts of the same family

\section{Electron repulsion}

12.1 From the Hückel model to the Hubbard model 256

12.1.1 The delocalised picture of $\mathrm{H}_{2} \quad 256$

12.1.2 The localised picture of $\mathrm{H}_{2} \quad 260$

12.1.3 From the molecule to the solid state 266

12.1.4 Application to one-band systems 269

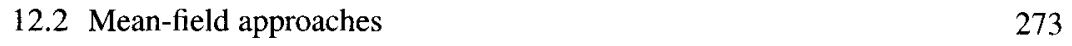

$\begin{array}{ll}\text { 12.2.1 The many-body problem } & 273\end{array}$

12.2.2 The Hartree-Fock method 274

12.2.3 Density functional theory 281

$\begin{array}{ll}12.3 \text { Conclusion } & 287\end{array}$

Solutions for exercises $\quad 291$

Appendix: Character tables $\quad 342$

$\begin{array}{ll}\text { Index } & 345\end{array}$ 